\title{
Raman Spectroscopic and Advanced Signal Processing analyses for Real Time Standoff Detection and Identification of Explosives
}

\section{Adel Abdallah}

College of Military Engineering

Alaaeldin Mahmoud

College of Military Engineering

\section{Mohamed Mokhtar \\ Military Technical College}

\section{Aiman Mousa}

Military Technical College

Yahia Elbashar ( $\nabla$ y_elbashar@yahoo.com )

Cairo University https://orcid.org/0000-0002-1362-2189

\section{H. S. Ayoub}

Cairo University

\section{Research Article}

Keywords: Raman spectroscopy, Signal processing, Pearson correlation, Explosives detection, Standoff detection

Posted Date: April 21st, 2021

DOI: https://doi.org/10.21203/rs.3.rs-426964/v1

License: (9) This work is licensed under a Creative Commons Attribution 4.0 International License. Read Full License 


\section{Abstract}

Laser Raman spectroscopy is a powerful instrument commonly used for detection of bulk and trace amounts of explosives. The work carried out in this paper is divided into two phases; the first phase is to propose a real time standoff explosive detection and identification system based on Raman spectroscopy that can be deployed in static checkpoints. The measurement is performed for samples placed in contact and at distances up to 1 meter in ambient light conditions. The second phase is to propose a novel sophisticated signal processing and pattern recognition techniques for accurate identification and classification of the investigated materials.

\section{Introduction}

Real time detection and identification of explosives become an urgent need to thwart the increasing terroristic operations. This requires standoff detection techniques that can provide instant detection and classification of the materials and avoid the necessity for direct contact with the investigated material or the preparation of a sample for detection. One of the most promising techniques for this purpose is the Raman spectroscopy that can provide remote detection and classification of the materials with high sensitivity. Raman spectroscopy has been recognized as a powerful technique capable of detecting and identifying a large variety of biological, chemical or explosive materials which considered as a threat for our humanity from terrorist attacks. Raman spectra of different molecules have their unique and specific shape characteristics that can be selected for distinction between different types of explosives [1, 2]. Different detection systems based on exploiting Raman effect for detection and identification of explosives or spectroscopic analysis for instrumental techniques have been developed and introduced [335]. In [18] Raman spectra of different chemical materials over a long distance up to $54 \mathrm{~m}$ were successfully recorded for explosive identification. Most of the signatures could be well recognized despite some missing information, including the shifts and loss of some peaks of the observed Raman signal. Researchers in [17] obtained the Raman signal intensities of ammonium nitrate, potassium nitrate, and sodium nitrate in solid samples in a distance range from $2 \mathrm{~m}$ to $10 \mathrm{~m}$. Although the Raman spectra of these three samples look similar in the highest peak; small differences can serve as a signature for discriminating between them. Remote Raman spectra of naphthalene, sulfur, nitrobenzene, ammonium nitrate, potassium chlorate and urea is measured from $1752 \mathrm{~m}$ distance during daylight using spontaneous Raman signals with integration time of $1-30 \mathrm{~s}$ with high $\mathrm{S} / \mathrm{N}$ ratio. The work is performed as attempt to use these systems to work from platforms mounted on helicopters and light airplanes [19]. The UV resonance Raman spectroscopy for of TNT, PETN, RDX, HMX is measured at 204-257 nm and it was found out that the spectral characteristics of the Raman signal increases and improved significantly with the decrease of excitation laser wavelength [20]. Recently, an experiment was conducted using a novel Raman wide field imaging spectrometer for detecting explosive residues operating at UV spectrum showed the advantages of using this spectrum over the Infrared systems [21]. Most of the previous work in this area of research focused on the detection process of the extremely weak Raman backscattered signal. As a result of these studies, detecting explosives remotely or generally at further distance is 
challenging and requires choosing appropriate laser excitation wavelength, increasing the laser output power, using a telescope with optics optimized for this purpose to collect the weak backscattered Raman signal, using a detector with enhanced sensitivity, and using longer integration time, and or nanosecond time gating capability or generally reducing the ambient noise to enhance the signal to noise ratio. The mathematical model that describes the process of Raman effect was introduced in many references such as $[22,23]$. Laser light is electromagnetic radiation that contains an oscillating electric field. Raman scattering arises when a laser light is incident on a material causes molecular vibrations and distortion of the electron cloud or change in the polarizability of the molecule. Polarizability is determined by the ability of the electron cloud to interact with an incident electric field. The intensity of the Raman scattering depends on the excitation laser power, the square of the polarizability of the investigated molecule and inversely proportional to the fourth power of the wavelength of the excitation laser. Thus, the molecular information that distinguishes certain substance from other substances depends on one molecular property which is the polarizability, while the laser power and wavelength can be controlled by the designer or the user $[22,23]$. When a laser source with proper power and wavelength illuminates a material, there will be different types of scattered radiation. When laser light is scattered by a material, almost all of the scattered photons are due to an elastic process or the Rayleigh scattering in which the scattered photons have the same wavelength as that of the incident light. On the other hand, very small amount of the scattered photons is due to inelastic process in which the scattered photons have different wavelength from the incident light. The inelastic scattering process was first experimentally observed by Raman in 1928. These scattering processes are illustrated in Figures 1, and 2. In Rayleigh scattering process, an electron in the ground state is excited and relaxes to the original ground state releasing a scattered photon with the same wavelength. Raman scattering process is classified into Stokes Raman scattering and anti-Stokes Raman scattering. In Stokes Raman scattering process, an electron is excited from the ground state to a virtual energy state and relaxes to a vibrational state. In the Stokes Raman scattering, energy of the incident photons is transferred or absorbed by the molecule thus in this case, the scattered photon has less energy or a longer wavelength than the wavelength of the incident photon. In Anti-Stokes Raman scattering process an electron is excited from the vibrational state to a virtual state and relaxes to the ground state. In this case energy is transferred from the molecule to the incident photon causes the scattered photon to have a shorter wavelength. This difference in energy or the shift in wavelength is corresponding to the energy of vibrational modes of the molecule which can be regarded as a fingerprint that uniquely identifies the chemical structure of the sample. The Rayleigh scattering process is the dominant, while the Raman scattered energy is extremely weak such that only one photon scatters in every $10^{6}$ to $10^{8}$ photons. Raman spectrum is expressed in the form of the intensity of the scattered photons versus wavenumber that represent the Raman shift in $\mathrm{cm}^{-1}$ and can be calculated from:

Raman shift $\left(\mathrm{cm}^{-1}\right)=\left(\frac{1}{\lambda_{o}}-\frac{1}{\lambda_{s}}\right)$ 
Where $\lambda_{o}$ is the laser excitation wavelength and $\lambda_{s}$ is the Raman shifted wavelength, both in $\mathrm{cm}$.

One possible solution to the problem of the low signal intensity obtained from Raman spectroscopy is the surface enhanced Raman spectroscopy (SERS) [24-28], and surface-enhanced resonance Raman scattering (SERRS) [29]. SERS enhanced the Raman signal to noise ratio dramatically. On the other hand, detection using SERS technique needs sample preparation, fixed sample, and the SERS spectra sometimes is different from a normal Raman spectrum for the same material, so generally it can't be used practically for standoff detection of explosives in real time, and/or to detect traces on moving objects. Building real-time explosive material identification analysis software is more demanded by Raman spectroscopy. These systems require a robust signal processing built for signature matching for accurate identification of the explosive materials. Pattern recognition technique of principal component analysis (PCA) with Raman detection systems was proposed in [15]. Some techniques were used to extract and classify the Raman signature are presented in [30,31]. The main contribution of this paper is not measuring the Raman spectra of different explosive materials at long distances as this has already been discussed and introduced in the literature but to propose an integrated tool for explosive detection and identification that facilitates the analysis process of different bulk or traces of explosive substances based on advanced signal processing to be used as a reliable standoff checkpoint explosive detection and identification system. In this paper we adopt a novel signal processing technique that provides faster, and more accurate results which are required for real time computations compared to the other techniques. The proposed framework stores the processed acquired backscattered Raman signal and constructs an equivalent material signature using a compaction method to build up material's signatures library. In the material detection phase, signature matching process carried out between the formed signature for the investigated material and the stored signatures in the constructed library. The proposed framework under normal conditions detects the material under test within few milliseconds as a result of library fetching optimization. The proposed models are implemented using MATLAB codes that allow the operator to get real time detection of explosives and easy to use recognition interface form shows the identified substances, their classification, the signature plot, the matching level of the investigated substance, and gives threat identification with text and audio alarm. The tested explosive materials are trinitrotoluene (TNT), pentaerythritol tetranitrate (PETN), cyclotetramethylene-tetranitramine (HMX), ammonium nitrate-fuel oil (ANFO), Cyclotrimethylenetrinitramine (RDX), and Ammonium Nitrate.

\section{Experimental Setup And Components Description}

In this study, selected conventional explosives materials are tested such as TNT, PETN, HMX, ANFO, and Ammonium Nitrate. Some safe materials such as sugar, salt, and flour are also studied to test the system's ability to distinguish between explosives and safe materials. The system can be upgraded easily to include any other types of explosives. At the beginning, a Raman sample holder (Ocean Optics-RIP-PA$\mathrm{SH}$ ) that allows a working distance of $7.5 \mathrm{~mm}$ between the excitation laser source and the sample is used. The laser source beam is focused on the samples; the backscattered light from the sample is detected, and the Stokes-Raman spectra of the investigated materials are extracted and analyzed in the 
range $200-2000 \mathrm{~cm}^{-1}$. Detecting the explosive materials in contact is useful to allow storing the Raman signatures of the materials under test to build the required material library to be used in comparison with the materials to be identified later (explosive - safe - organic material...). The main system components and the setup of the experiment for detecting the explosive materials in contact and for stand-off detection up to $1 \mathrm{~m}$ are shown in Figure 3. It was reported that the environmental conditions could change the characteristic signals of explosives [2, 32]. In this experiment Raman spectrum of each of the investigated materials is collected at least 40 times in the lab and outside the lab in different days and different times during the days, this leads to increasing the probability of detection of the explosives in different environmental conditions. This is to account for any slight change in the Raman spectrum of the material under test due to the environmental change like room temperature, pressure, humidity, ambient light conditions, especially as the temperature change causes spectral variance. To study the variability and the stability of the collected Raman spectra and generally the effect of the background materials on the detection process, the experimental measurements are performed when the investigated bulk/trace materials is deposited on different background materials like polytetrafluoroethytene sheet (PTFE), sand, clay, traces on fingers and door handle. After building the material library, a variable beam expander with $2 x$ to $12 x$ expansion ratio is used to control the size of the laser spot at the sample to allow detecting the explosive materials at distance up to $1 \mathrm{~m}$. The Raman spectra of the materials are obtained using the microcontroller-controlled Ocean Optics QE65000 spectrometer. The QE65000 spectrometer has a 1200 line/mm grating and the detector used is a scientific-grade, back-thinned, TE Cooled (TEC), $1044 \times 64$ element CCD array from Hamamatsu (product number S7031-1006) with spectral range from UV to NIR (200-1100 nm). The spectral resolution of the spectrometer is $1.3 \mathrm{~nm}$. The used laser source for Raman excitation (LASER-785-LAB-ADJ-SMA) is a stabilized laser diode with Raman excitation wavelength of $785 \mathrm{~nm}$, adjustable output power $>350 \mathrm{~mW}$, beam divergence $0.5 \mathrm{mrad}$, spectral linewidth< $0.15 \mathrm{~nm}$ (FWHM), and SMA 905 fiber connection. It has integrated laser driver and is thermoelectrically cooled for optimum performance. The laser power is set to $400 \mathrm{~mW}$ in the experiment. The distance between the telescope and the Raman probe is $2 \mathrm{~cm}$. The variable beam expander is adjusted to provide 10x magnification and the measured laser spot size on the sample at $1 \mathrm{~m}$ is $1 \mathrm{~cm}$. The used Raman coupled fiber probe (Ocean Optics-RIP-RPB-785-SMA-SMA) supports an excitation wavelength of $785 \mathrm{~nm}$. The core diameters of the excitation fiber and the collection fiber are $105 \mu \mathrm{m}$ and $200 \mu \mathrm{m}$, respectively, both has NA of 0.22 , and SMA 905 connectors for excitation and collection fibers. The probe is characterized by the strong signal collection and high optical density laser line filtering (a notch filter at $785 \mathrm{~nm}$ (OD 6) is used to block the Rayleigh backscattered photons). The spectrometer interfaces to PCs through USB 2.0. A computer is used for signal processing and display of the results as will be explained in the next section. For all measurements performed in this experiment, the integration time used is $100 \mathrm{~ms}$.

\section{Signal Processing}

During this work part, the acquired Raman backscattered signal should be processed and recognized to identify the material under test (MUT). The MUT identification module should decide whether the material 
is safe, organic or explosive material with a probabilistic guess of the material type. The signal processing portion subdivided into two main phases. The training phase (material library construction) and the evaluation (engagement) phase. During the training phase, a set of identified materials should be fed to the training module to build the materials library. The second phase is the test or engagement phase where an unknown material should be investigated for safety. The proposed framework block diagram is shown in Figure 4.

Signal acquiring and processing are performed through the following steps:

\subsection{Raman Signal Acquisition}

After illuminating the MUT with the laser source, the backscattered Raman signal acquired by the Raman architecture setup, the data collected and formatted within a file that autonomously saved in a predefined location. After reading the material file, a preconditioning process should start to prepare the acquired information to be recognized.

\subsection{Acquired Signal Preconditioning}

The preconditioning process should include filtration, scaling and normalization. Filtration aims to improve the signal to noise ratio by removing the superimposed noise for maximizing the recognition rate and minimizing ambiguity. Scaling and normalization sub processes aim to unify the scale of all the acquired backscattered Raman signals from different materials to the same magnitude scale.

\subsection{Training Phase}

During the training phase, the proposed framework stores the processed acquired backscattered Raman signal and constructs an equivalent material signature using a compaction method to build up a material's signatures library. This library should be the recognition reference for the proposed framework. The trainer interface form is shown in Figure 5. The following subtasks are performed in the training phase:

\subsubsection{Supervised Training}

Through supervised training, the proposed framework learns the categories and the types of the materials under supervision of system operator. The operator feeds the system with a predefined material data, a material signature constructed from the fed data and further the operator feeds the system with the material category and type that sticks with the material generated signature.

\subsubsection{Signature Formation}

Material unique signature formed by compacting the acquired preconditioned backscattered Raman signal into constant width signature vector that describe the material unique pattern. Signature formation through compaction can mask different variations for the same material that may lead to false detection or recognition. 


\subsubsection{Library Construction (Patch Processing)}

This step builds up the material library by loading all the backscattered Raman signal files of specific material in patch mode, the system trainer give it a name and category. Material Library collects all the trained materials signatures in an organized form to facilitate signatures retrieval and matching. The library organized according to material category and material type to patches to speedup the matching subprocess.

\subsubsection{Library Control}

Constructed library comprises some features and functions as appending new signatures, modifying, deletion or even removing the whole signatures library.

\subsection{Engagement Phase}

Through this phase, the proposed framework engaged in an investigation channel and start to recognize the materials that passes through this channel, correct recognition rate mainly depends on the number of materials got trained and the number of records of the same material under different conditions and circumstances. The recognition interface form is shown in Figure 6. The following subtasks are performed in the engagement phase:

\subsubsection{Signature Matching}

At this step, a matching process carried out between the formed signature for the material under investigation and the trained signatures stored in the constructed library. The matching process uses Pearson correlation to achieve a quantitative matching criterion as follow:

$$
r_{x y}=\frac{\sum_{i=1}^{n}\left(x_{i}-\bar{x}\right)\left(y_{i}-\bar{y}\right)}{\sqrt{\sum_{i=1}^{n}\left(x_{i}-\bar{x}\right)^{2}} \sqrt{\sum_{i=1}^{n}\left(y_{i}-\bar{y}\right)^{2}}}
$$

Where $r_{x y}$ represents the correlation coefficient, $x$ represents the material under investigation signature vector, represents the library signature record.

\subsubsection{Autonomous Sensitivity Adjustment}

The proposed framework does not depend on a perfect matching between the investigated material signature and the stored signatures within library. The signature matching could be carried out using a predefined sensitivity level or it could be set to autonomously adjust (sensitivity scan) the sensitivity level until a stored library signature match the investigated signature at certain sensitivity level.

\subsubsection{Real Time Detection}


The proposed framework under normal conditions and opened sensitivity adjustment detects the MUT within few milliseconds as a result of library fetching optimization that could be considered real time recognition.

\subsubsection{Visual Signature Comparison}

The proposed framework includes a visual representation of the investigated material signature (Signature Curve) in addition to the recognized probable materials signatures to support the operator's decision making.

\subsubsection{Detection Alarming}

In case of detecting a dangerous or threat material, the proposed framework send out a warning siren and visual message that warn the operator about a probable threat material, in addition to its probable type and its probability ratio with respect to the adjusted sensitivity.

\section{Experimental Results And Analysis}

Performing the experiment during the daytime outside the lab up to $1 \mathrm{~m}$ to the sample using the current components and setup, we get a noisy Raman signal for each material but the detected signal still has its distinguished peaks of the Raman spectrum of the material. If the distance to the sample under test is increased beyond $1 \mathrm{~m}$, the signal to noise ratio is degraded. Improving the signal to noise ratio may be accomplished generally by choosing system components with better specifications such as laser power, wavelength, optics of the telescope, increasing the integration time, and using some techniques like nanosecond time gating. All the obtained Raman spectra, corresponding to the different samples under test, are saved in the library to be compared later with the measured samples. The proposed GUI and the written code allow saving any kind of explosive or other substances. When the measured sample is tested and its Raman spectrum is matched with any of the previously saved Raman spectrum of the explosive materials, alarm is initiated and the GUI shows the type of detected explosive material and the percentage of the matching level. The measured Raman spectra of the TNT, PETN, HMX, ammonium nitrate, ANFO, and RDX samples covering a range from 200 to $2000 \mathrm{~cm}^{-1}$ measured at $1 \mathrm{~m}$ as shown in Figures 7, 9, 11, 13,15 , and 17 respectively. It can be seen that all major peaks of the investigated substances can be identified in the obtained spectrum at 1 meter distance. The Raman spectrum is recorded and then compared to the stored Raman spectra in the material library for matching and identification. The result of investigating the TNT sample is obtained and displayed on the recognition interface form shown in Figure 8. The TNT material is identified and classified as "Explosive Material" with matching level of $98.77 \%$. This shows that the proposed system could correctly and accurately identify the TNT material. Following the same steps, the identification results of the PETN, HMX, ammonium nitrate, ANFO, and RDX are shown in Figures 10,12,14, 16, and 18 with matching levels of $97.41 \%, 95.64 \%, 99.93 \%, 97.2$, and 98.92 respectively. After some days and testing other samples of PETN, and HMX, the system could identify the materials but with lower matching levels that was $79.83 \%$, and $67.51 \%$, respectively. For these 
samples of PETN and HMX, the low matching level could be attributed to the sample itself (impurity, humidity of the sample, crystallity of the sample), in addition to the environmental conditions in the lab. Also, this can be attributed to the laser heating effect on the exposed sample which can change its chemical structure during the analysis. Morphology of the sample (size and shape) and cyrstallinty can affect strongly the obtained spectra. So, separate research should be conducted to study these effects on the sample's Raman spectra but this is not our goal in this study. Thus, it is sufficient for the current work this matching efficiency at this stage and the ability of the system to identify the material.

The obtained Raman spectrum from an investigated sample whether it is pure substance or composed of mixture of two or many substances will contain the Raman information about all of the molecules of the substances that form the sample. As the laser scans the examined area of the mixture, there will be some characteristic peaks that distinguish the components of the mixture. To extend the system capabilities,

the system is tested to detect the explosives that are mixed with safe materials. To show this capability, a sample of a mixture contains $40 \% \mathrm{HMX}, 30 \%$ flour, and $30 \%$ sugar is investigated. The measured Raman spectrum of the mixture is shown in Figure 19. The proposed system could detect the safe and the explosive substances and the identification results show approximately equal matching levels, as shown in Figure 20.

The close match between sugar and flour is due to their close chemical structure as they have the same heterocylic structure. $\mathrm{HMX}$ shows quite significant difference from them because the presence of $\mathrm{N}-\mathrm{NO}_{2}$ bond which is neither in sugar nor on flour

To confirm the ability of the system to detect the explosives mixed with safe substances, another sample consists of $50 \%$ TNT, and $50 \%$ sugar is tested. The measured Raman spectrum of the mixture of the sugar and the TNT is shown in Figure 21. Figure 22 shows the ability to identify the TNT and the sugar with matching levels of $84 \%$, and $82.24 \%$, respectively.

The data in the figures presented in this section illustrate that the proposed Raman system can accurately identify the investigated substances. Regardless the obtained matching level, any indication of the presence of explosive is important to take actions in time, and generally in this case the probability of miss is unacceptable.

\section{Conclusion}

In this paper, we proposed a real time standoff detection and identification of explosives based on high performance Raman spectroscopy and novel signal processing technique. The results presented in this paper show the ability of the proposed Raman system to detect, identify the investigated substances, and classify them to safe and explosives substances. The proposed models are implemented using MATLAB codes that allow the operator to get real time detection of explosives and easy to use recognition interface form shows the identified substances, their classification, and the signature plot. The investigated substances are TNT, PETN, HMX, ammonium nitrate, ANFO, and RDX that could be identified 
with matching levels $98.77 \%, 97.41 \%, 95.64 \%, 99.93 \%, 97.2$, and 98.92 , respectively. The measurement is performed at distances up to 1 meter in ambient light conditions. It is also shown the ability of the proposed system to detect and differentiate between the explosive materials accurately even if it is mixed with other safe material which is one of the important results presented in this paper. This is demonstrated by investigating a sample contains $40 \%$ of $\mathrm{HMX}, 30 \%$ sugar, and $30 \%$ of flour, and another sample formed of $50 \%$ of TNT, and $50 \%$ of sugar to confirm the results. The system can be used as standoff check point explosive detection system and generally can be used for building a compact system for Homeland security and for securing the important facilities. In the future work we intend to study the identification of different new explosives, explosive mixtures and pbx; to find the trace limit of sample identification for the same distance $1 \mathrm{~m}$; and to study the main peak shift for example TNT under different conditions and different common mixtures.

\section{References}

[1] D. D. Tuschel and A. V. Mikhonin, "Deep ultraviolet resonance Raman excitation enables explosives detection," Appl. Spectrosc., vol. 64, pp. 425-432, 2010.

[2] G. Mogilevsky, L. Borland, M. Brickhouse, and A. W. Fountain, "Raman Spectroscopy for Homeland Security Applications," International Journal of Spectroscopy, vol. 2012, 2012.

[3] A. K. Misra, S. K. Sharma, D. E. Bates, and T. E. Acosta, "Compact standoff Raman system for detection of homemade explosives," Proc. SPIE, vol. 7665, 2010.

[4] J. C. Carter, S. M. Angel, M. Lawrence-Snyder, J. Scaffidi, R. E. Whipple, and J. G. Reynolds, "Stand-off detection of high explosive materials at 50 meters in ambient light conditions using a small Raman instrument," Appl. Spectrosc., vol. 59, pp. 769-775, 2005.

[5] M. Gaft and L. Nagli, "UV gated Raman spectroscopy for standoff detection of explosives," Optical Materials, vol. 30, pp. 1739-1746, 2008.

[6] S. Botti, S. Almaviva, L. Cantarini, A. Palucci, A. Puiu, and A. Rufoloni, "Trace level detection and identification of nitro-based explosives by surface-enhanced Raman spectroscopy," J. Raman Spectrosc. , vol. 44, pp. 463-468, 2013.

[7] S. R. Khandasammy, M. A. Fikiet, E. Mistek, Y. Ahmed, L. Halámková, J. Bueno, and I. K. B. Lednev, "Raman spectroscopy applications in forensic science," Forensic Chem., vol. 8, pp. 111-133, 2018.

[8] W. Zhang, Y. Tang, A. Shi, L. Bao, Y. Shen, R. Shen, and Y. Ye, "Recent Developments in Spectroscopic Techniques for the Detection of Explosives," Materials 2018, 11, 1364; doi:10.3390/ma11081364, vol. 11, 2018.

[9] N. John and S. George, "Spectroscopic Methods for Nanomaterials Characterization," presented at the Raman Spectroscopy, 2017. 
[10] A. Hakonen, et al., , "Detection of nerve gases using surface- enhanced Raman scattering substrates with high droplet adhesion," Nanoscale, vol. 8, pp. 1305-1308, 2016.

[11] A. e. a. Hakonen, "Explosive and chemical threat detection by surface-enhanced Raman scattering: A review," Anal. Chimica Acta., vol. 893, pp. 1-13, 2015.

[12] M. Marshall and J. C. Oxley, Aspects of Explosives Detection. Oxford: Elsevier, 2008.

[13] P. Wen, et al., , "Key challenges and prospects for optical standoff trace detection of explosives," Trends Anal. Chem. , vol. 100, pp. 136-144, 2018.

[14] G. J. Edelman, et al.," "Hyperspectral imaging for non-contact analysis of forensic traces," Forensic Sci. Int., vol. 223, pp. 28-39, 2012.

[15] J. Hwang, N. Choi, A. Park, J.-Q. Park, J. H. Chung, S. Baek, S. G. Cho, S.-J. Baek, and J. Choo, "Fast and sensitive recognition of various explosive compounds using Raman spectroscopy and principal component analysis," J. Mol. Struct., vol. 1039, pp. 130-136, 2013.

[16] B. Zachhuber, et al., , "Stand-off Raman Spectroscopy of Explosives," in Proc. of SPIE.2010, 2010, pp. 78380F-1 - 78380F-10.

[17] L. Zhang, H. Y. Zheng, Y. P. Wang, L. Ding, and L. Fang, "Characteristics of Raman spectrum from stand-off detection," Acta Phys. Sin., vol. 65, p. 054206, 2016.

[18] H. C. Jin and S. G. Cho, "Nanosecond Gated Raman Spectroscopy for Standoff Detection of Hazardous Materials," Korean Chem. Soc., vol. 35, pp. 3547-3552, 2014.

[19] C. Giardino, et al., , "Airborne hyperspectral data to assess suspended particulate matter and aquatic vegetation in a shallow and turbid lake," Remo. Sens. Environment., vol. 157, pp. 48-57, 2015.

[20] M. Ghosh, L. Wang, and S. A. Asher, "Deep-ultraviolet resonance raman excitation profiles of NH4NO3, PETN, TNT, HMX, and RDX," Appl. Spectrosc. , vol. 66, pp. 1013-1021, 2012.

[21] K. T. Hufziger, S. V. Bykov, and S. A. Asher, "Ultraviolet Raman wide-field hyperspectral imaging spectrometer for standoff trace explosive detection," Appl. Spectrosc., vol. 71, pp. 173-185, 2016.

[22] M. Lopez-Lopez and C. García-Ruiz, "Infrared and Raman spectroscopy techniques applied to identification of explosives," TrAC Trends Anal. Chem., vol. 54, pp. 36-44, 2014.

[23] A. W. Fountain, et al., , "Recent advances and remaining challenges for the spectroscopic detection of explosive threats," Appl. Spectroscopy, vol. 68, pp. 795-811, 2014.

[24] M. liszewska, B. Bartosewicz, B. Budner, B. Nasilowska, M.Szala, J. L. Weyher, I. Dzięcielewski, Z.Mierczyk, and B. J. Jankiewicz, "Evaluation of selected SERS substrates for trace detection of explosive materials using portable Raman systems," Vibrational Spectroscopy, 2018. 
[25] K. E. Brown, et al., , "Advances in explosives analysis - part II: Photon and neutron methods," Anal. Bioanal Chem., vol. 408, pp. 49-65, 2016.

[26] K. L. Gares, et al., , "Review of explosive detection methodologies and the emergence of standoff deep UV resonance Raman," J. Raman Spectroscopy, vol. 47, pp. 124-141, 2016.

[27] J. Yinon, Counter Terrorist Detection Techniques of Explosives. Amsterdam, Netherlands: Elsevier Science, 2007.

[28] M. Á. F. d. I. Ossa, CarmenGarcía-Ruiz, and J. M. Amigo, "Near infrared spectral imaging for the analysis of dynamite residues on human handprints," Talanta vol. 130, pp. 315-321, 2014.

[29] J. L. Gottfried, et al.,, "Strategies for residue explosives detection using laser-induced breakdown spectroscopy," J. Anal. Atom. Spectrom., vol. 23, pp. 205-216, 2008.

[30] P. M. Pellegrino, E. L. Holthoff, and M. E. Farrell, Laser Based Optical Detection of Explosives. FL, USA: Taylor \& Francis Group: Boca Raton, 2015.

[31] M. R. Almeida, et al.,, "Detection of explosives on the surface of banknotes by Raman hyperspectral imaging and independent component analysis," Anal. Chimica Acta., vol. 860, pp. 15-22, 2015.

[32] D. Tuschel, "Raman Thermometry," Spectroscopy Online, vol. 31, pp. 8-13, 2016.

[33] Hany S.Ayoub, Ashraf F.El-Sherif, Ahmed Elbeih, " Hyperspectral imaging and remote trace detection of cis-1,3,4,6-tetranitrooctahydroimidazo-[4,5 d] imidazole (BCHMX) compared with traditional explosives using laser induced fluorescence", Defence Technology, doi.org/10.1016/j.dt.2020.09.008, 2020

[34] Ahmed Mahfouz, Ahmed Emara, H.S.Ayoub, Ashraf.F.El-Sherif, Ahmed El Fatih, Y.H.Elbashar "Thermoluminescence spectroscopic analysis of fuel mixed cooking oil using Hyperspectral analysis", Journal of Optics, 49(3), 2020 , 370-383

[35] H. S. Ayoub , A. M. Mokhtar , Ashraf F. El-Sherif , Y.H.Elbashar, "Modified Range Equation for Exact Modeling and Design Optimization of Active Laser Remote Sensing Systems", Optical and quantum electronics, doi.org/10.1007/s11082-021-02759-5, (2021) 53:110

\section{Figures}




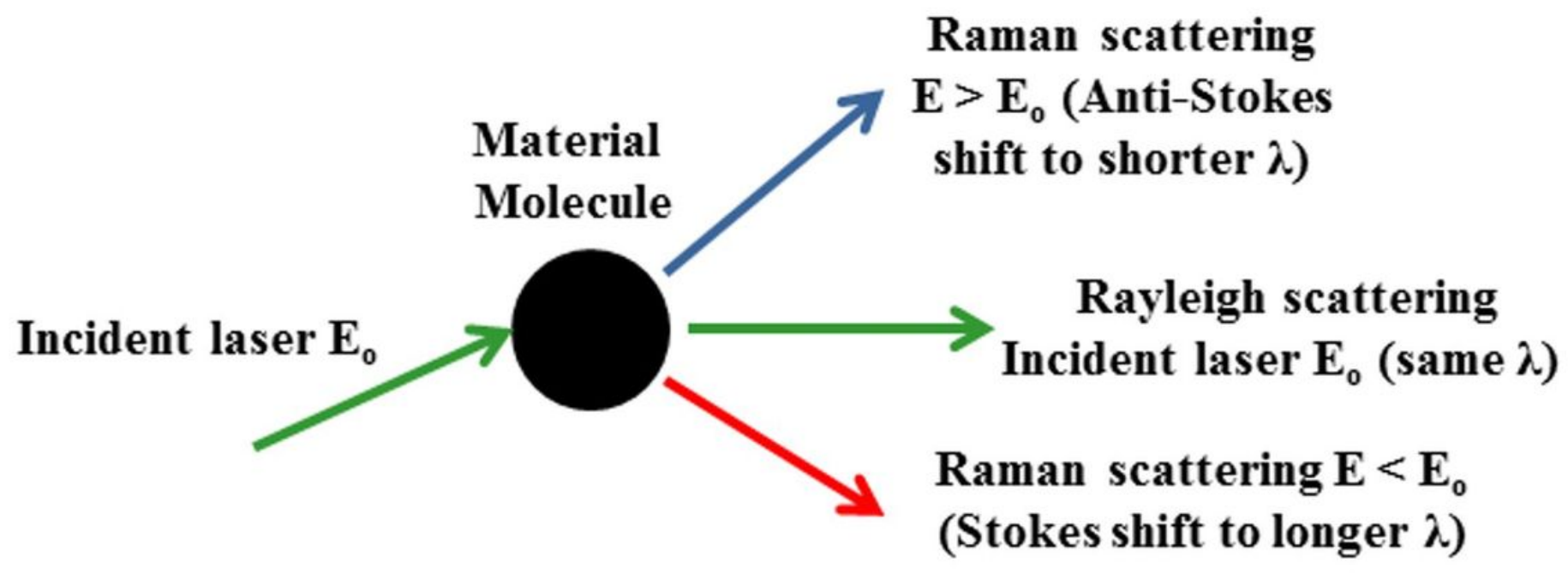

Figure 1

Diagram shows the Raman Scattering Processes

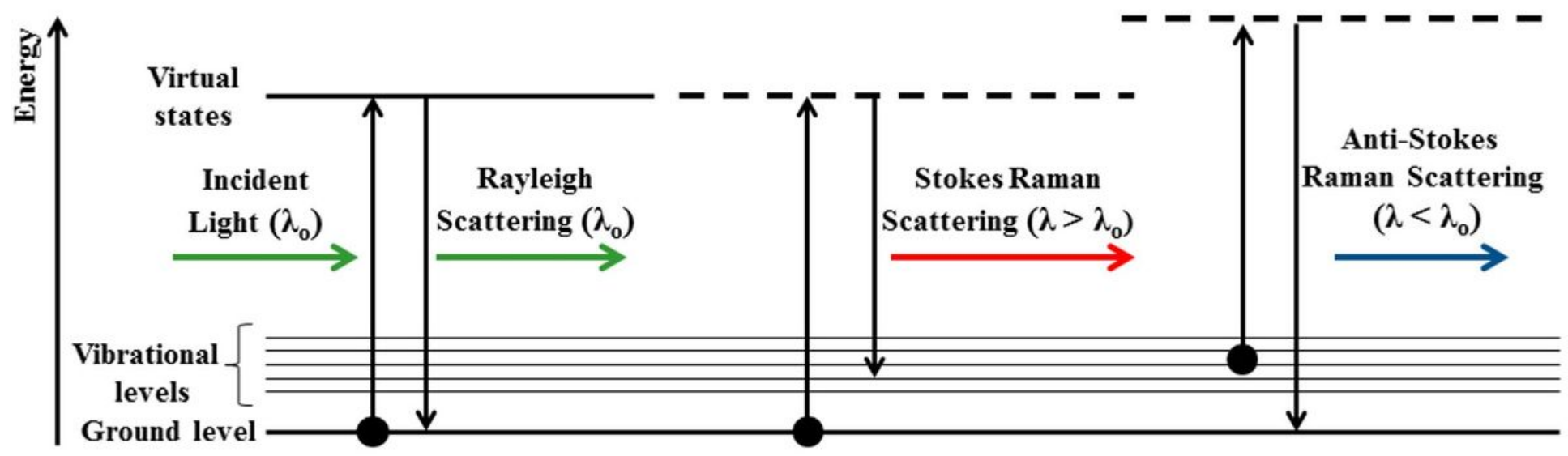

Figure 2

The energy diagram of Rayleigh and Raman scattering. 


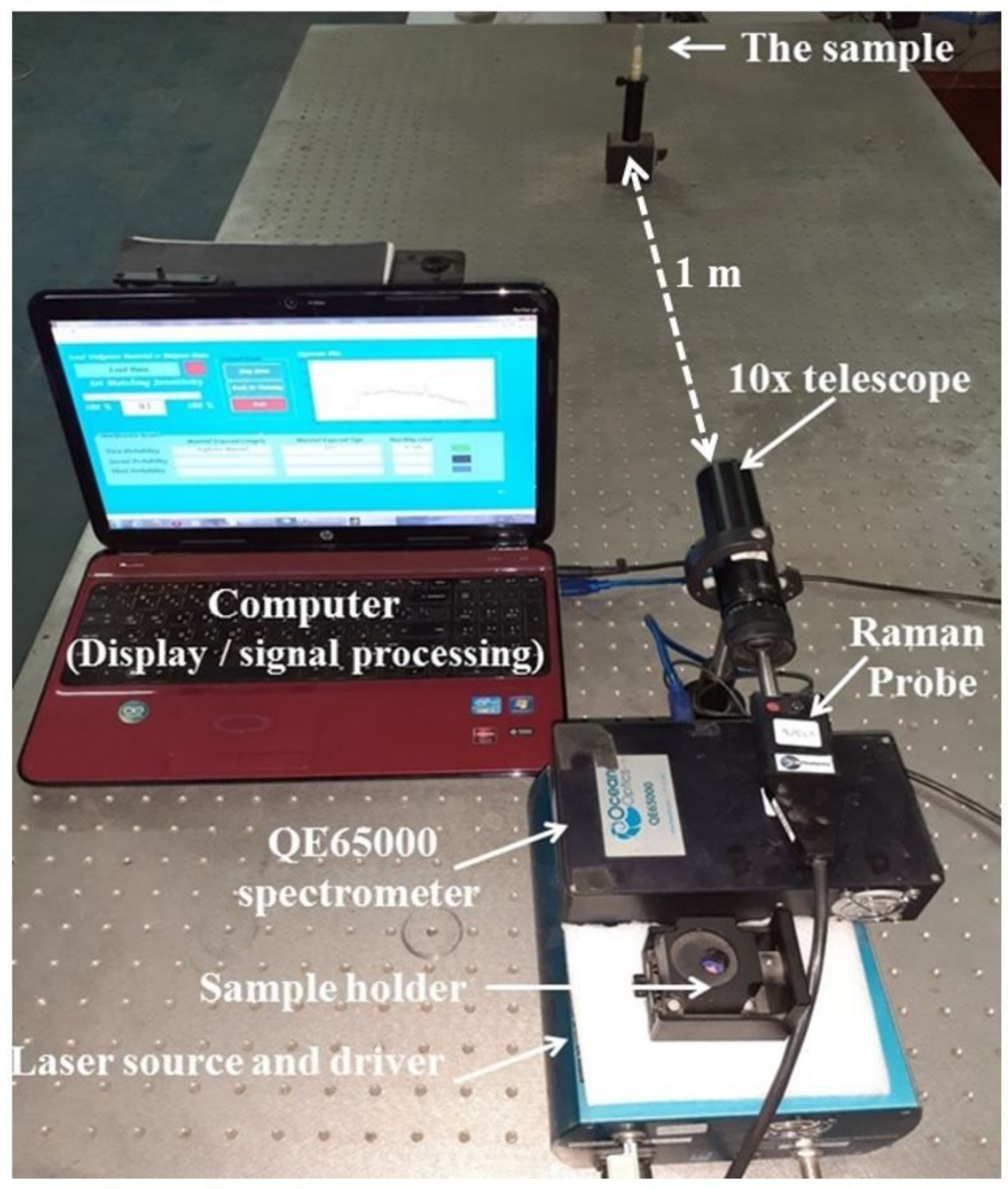

Figure 3

The experimental setup of the Raman system. 


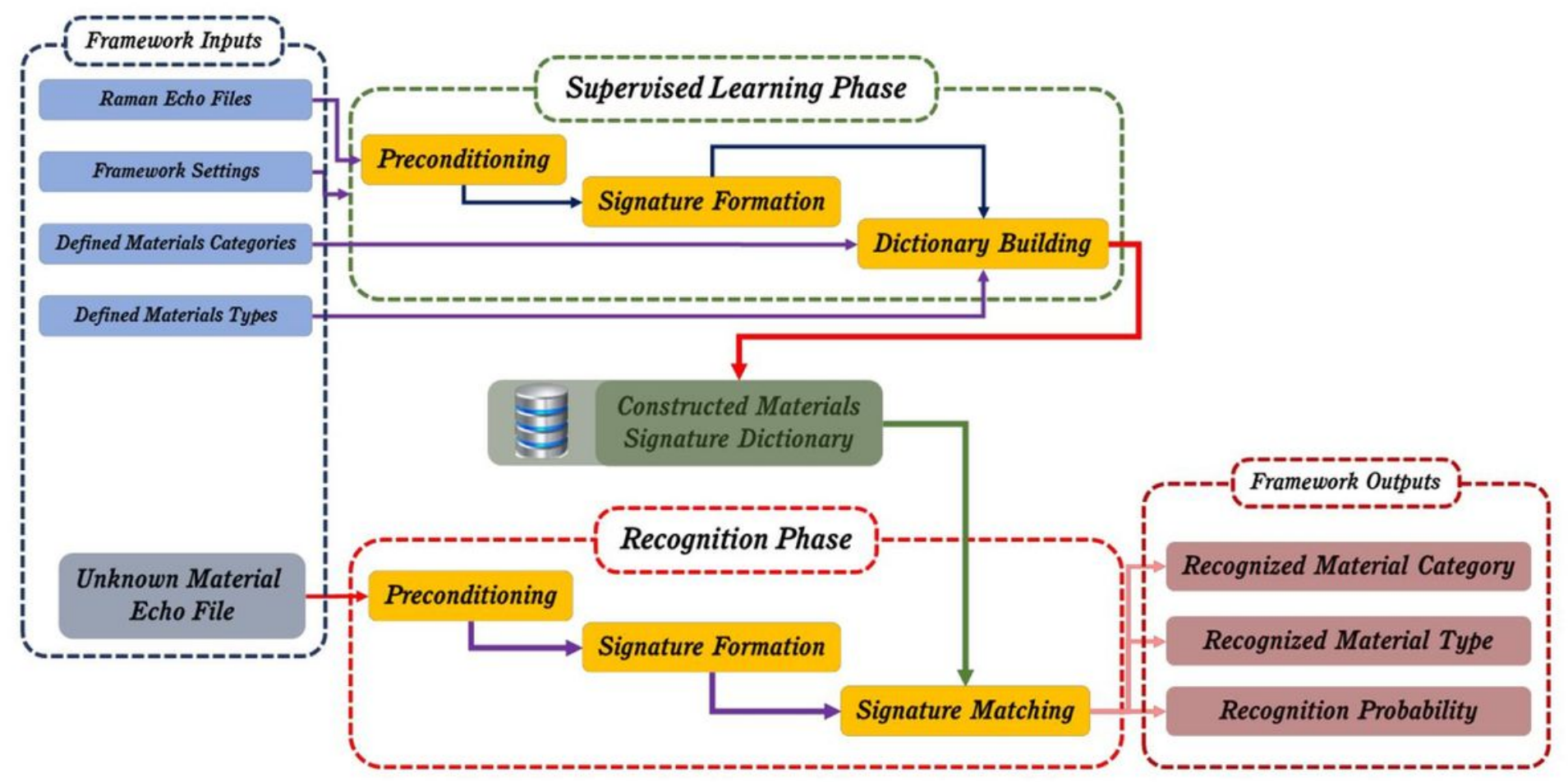

\section{Figure 4}

The proposed framework block diagram Signal acquiring and processing are performed through the following steps:

Step 1 : Load the material or mixture data from excel sheet

\section{Load Data}

Step 2: Choose material category (explosive-organic - safe)

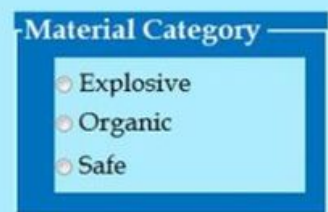

Step 3 : Assign a name for the material

New Material

elected

System Training

\section{Add Material Delete Material}

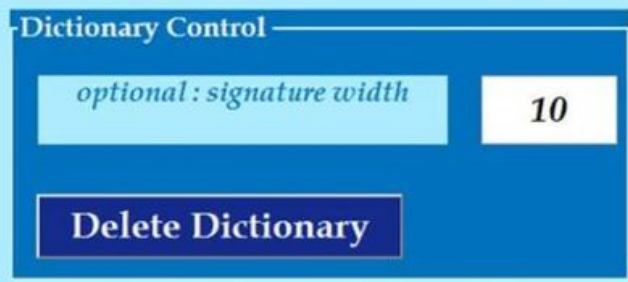

\section{System Test}

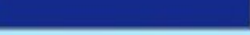

Delete Material 
Load Data

Set Matching Sensitivity

.

$100 \%$

$100 \%$
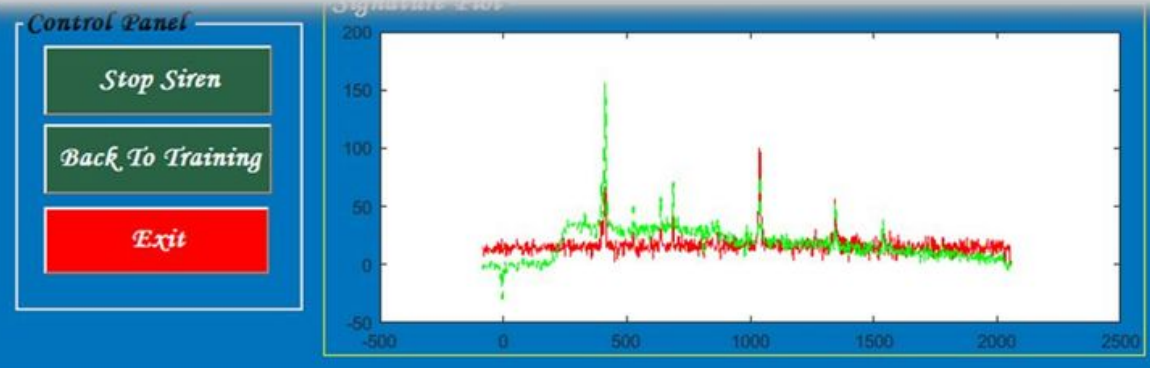

Identification Results

First Probabifity

Material Expected Category

Explosive Material

Second Probability

Third Probability
Material Expected Type

Amonium Nitrate

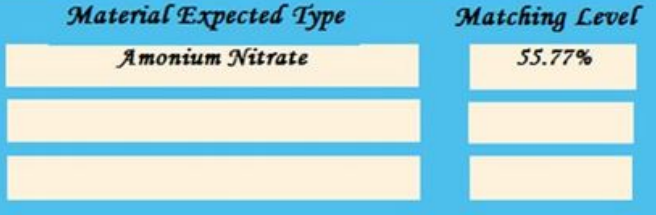

\section{Figure 6}

The recognition interface form.

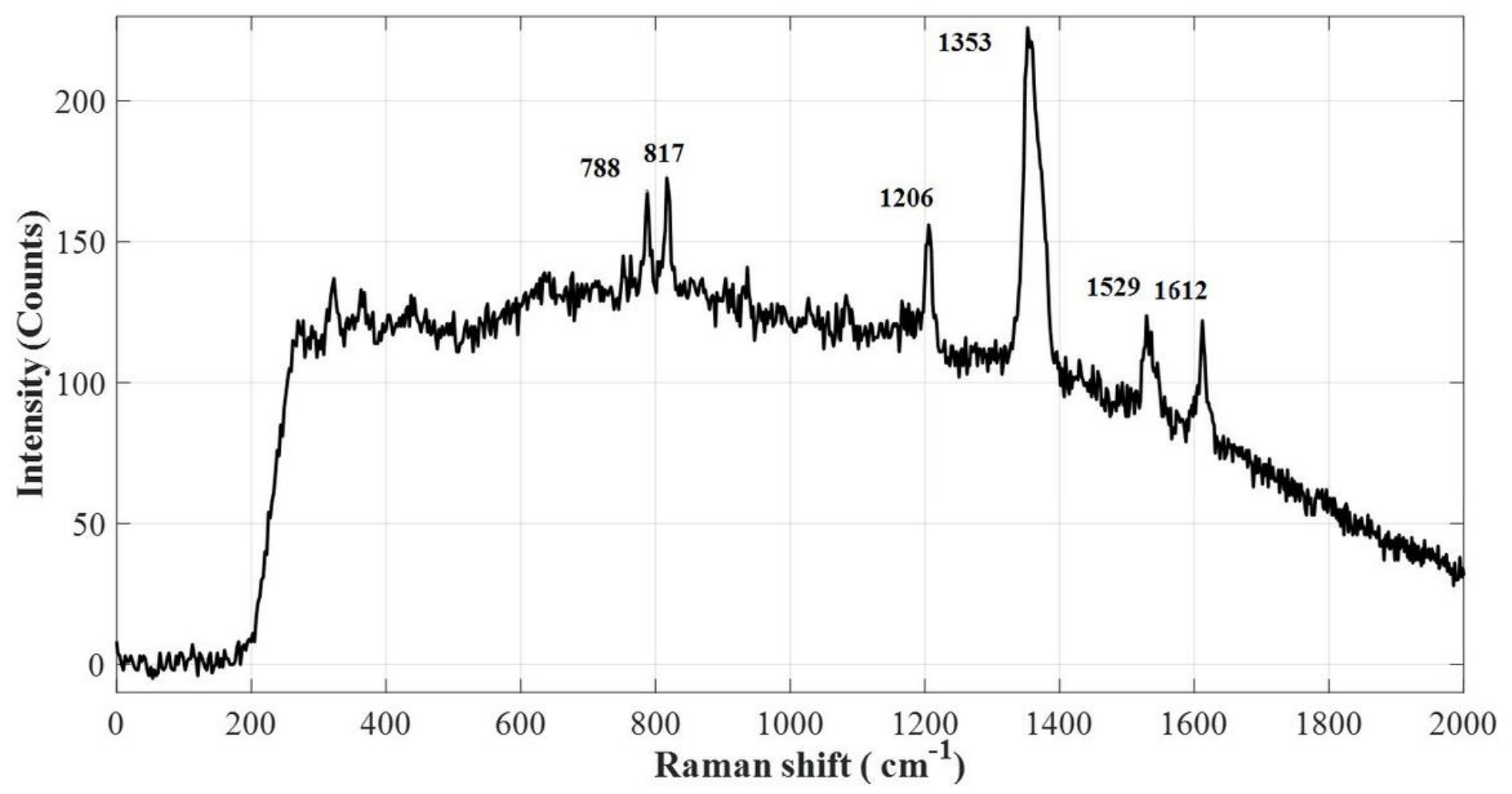

Figure 7

The measured Raman spectra of TNT sample placed at $1 \mathrm{~m}$ from the excitation laser source measured with $100 \mathrm{~ms}$ integration time 


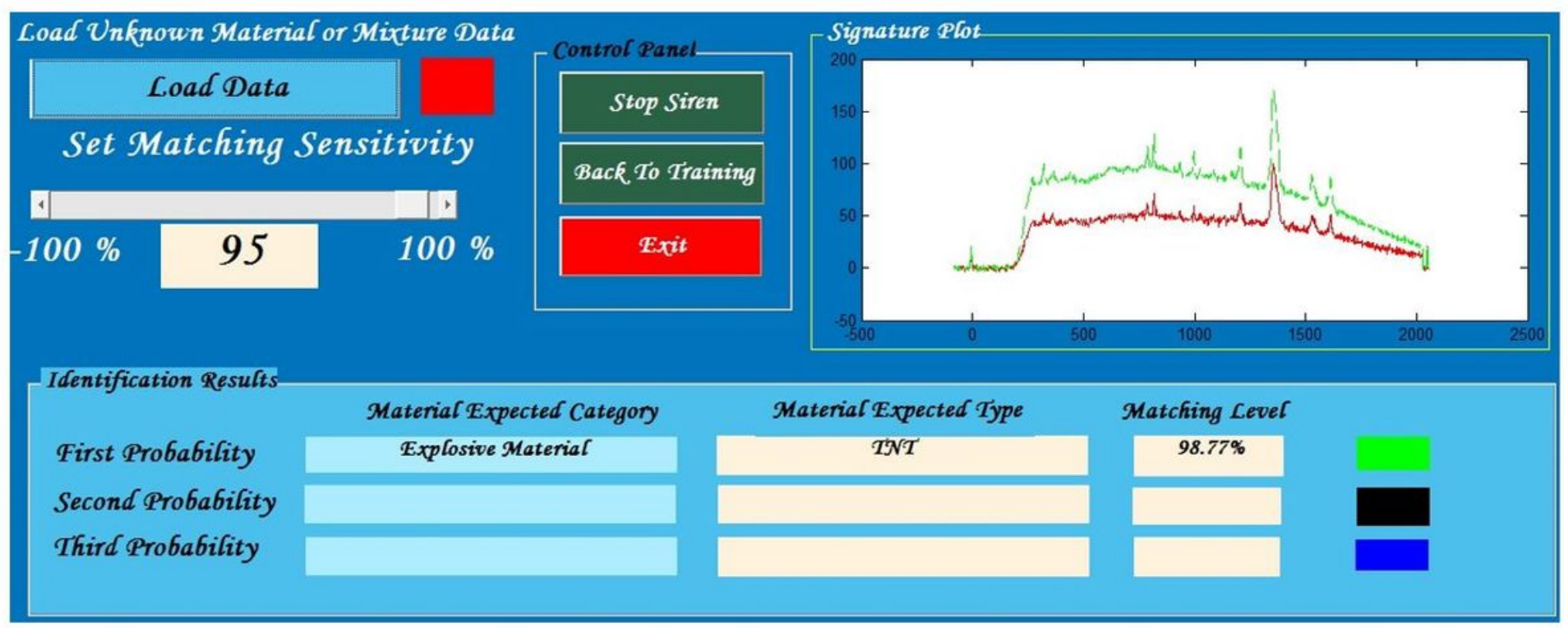

Figure 8

The recognition interface form shows that the material type is TNT with matching level of $98.77 \%$.

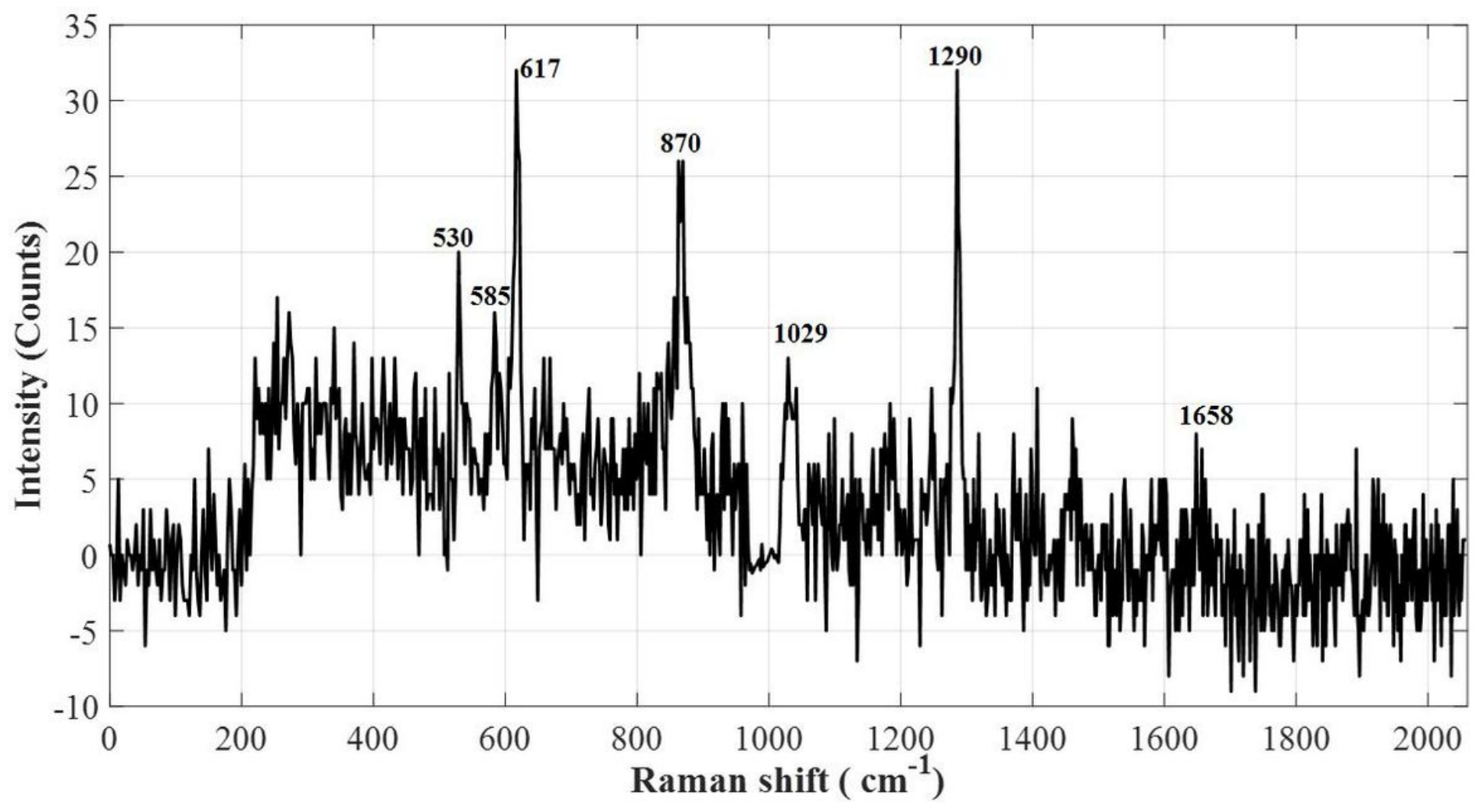

Figure 9

The measured Raman spectrum of the of PETN sample. 


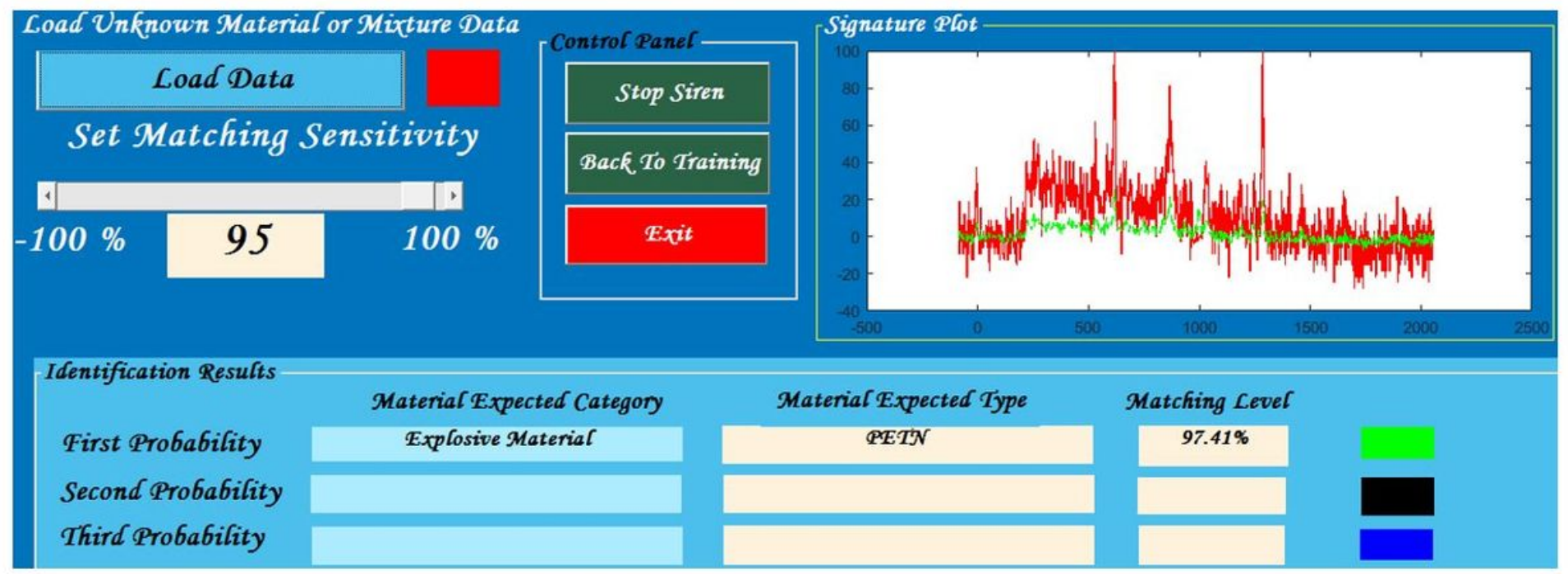

Figure 10

The recognition interface form shows that the material type is PETN with matching level of $97.41 \%$.

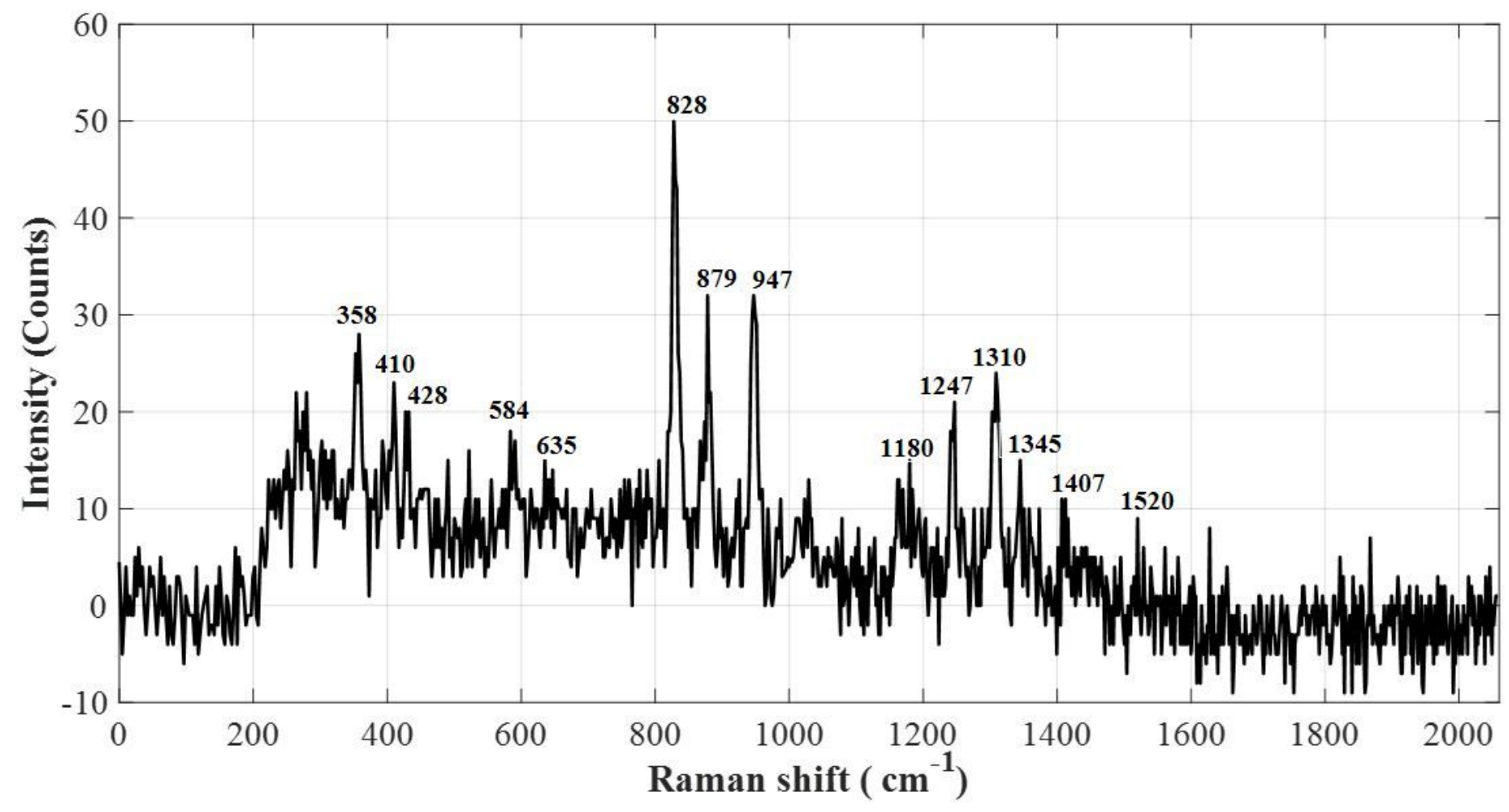

Figure 11

The measured Raman spectrum of the of HMX sample. 


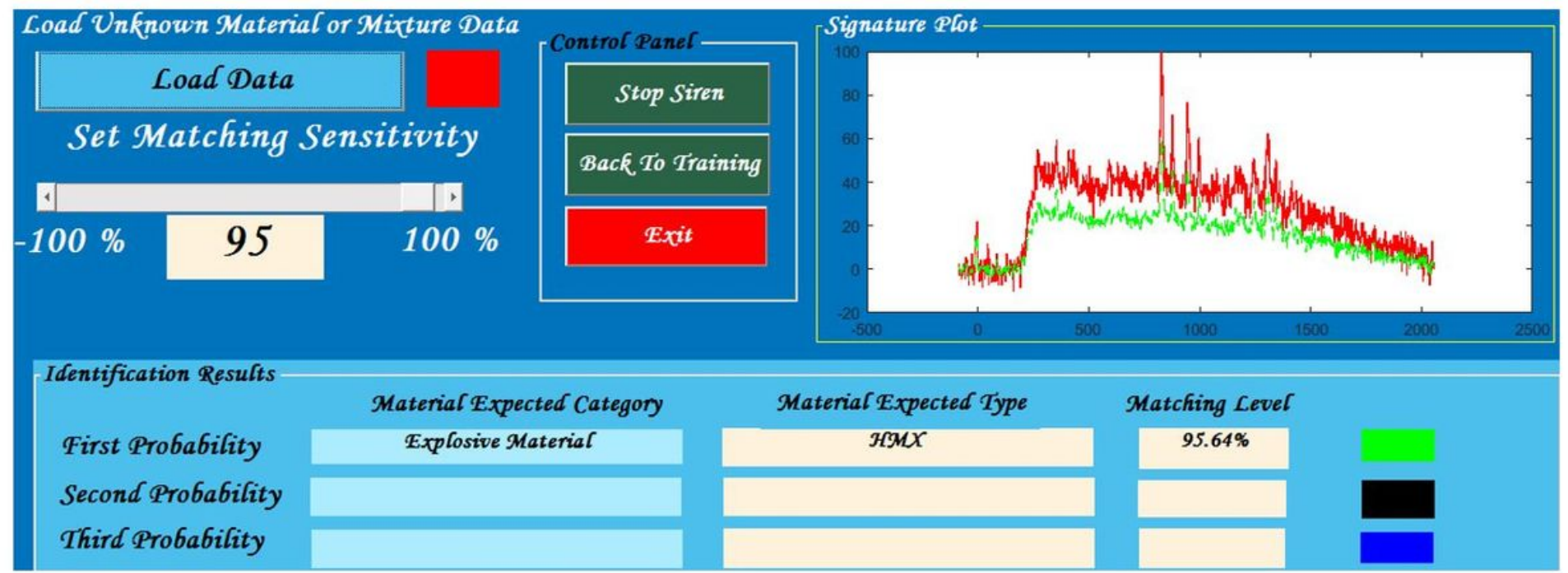

Figure 12

The recognition interface form shows that the material type is $\mathrm{HMX}$ with matching level of $95.64 \%$.

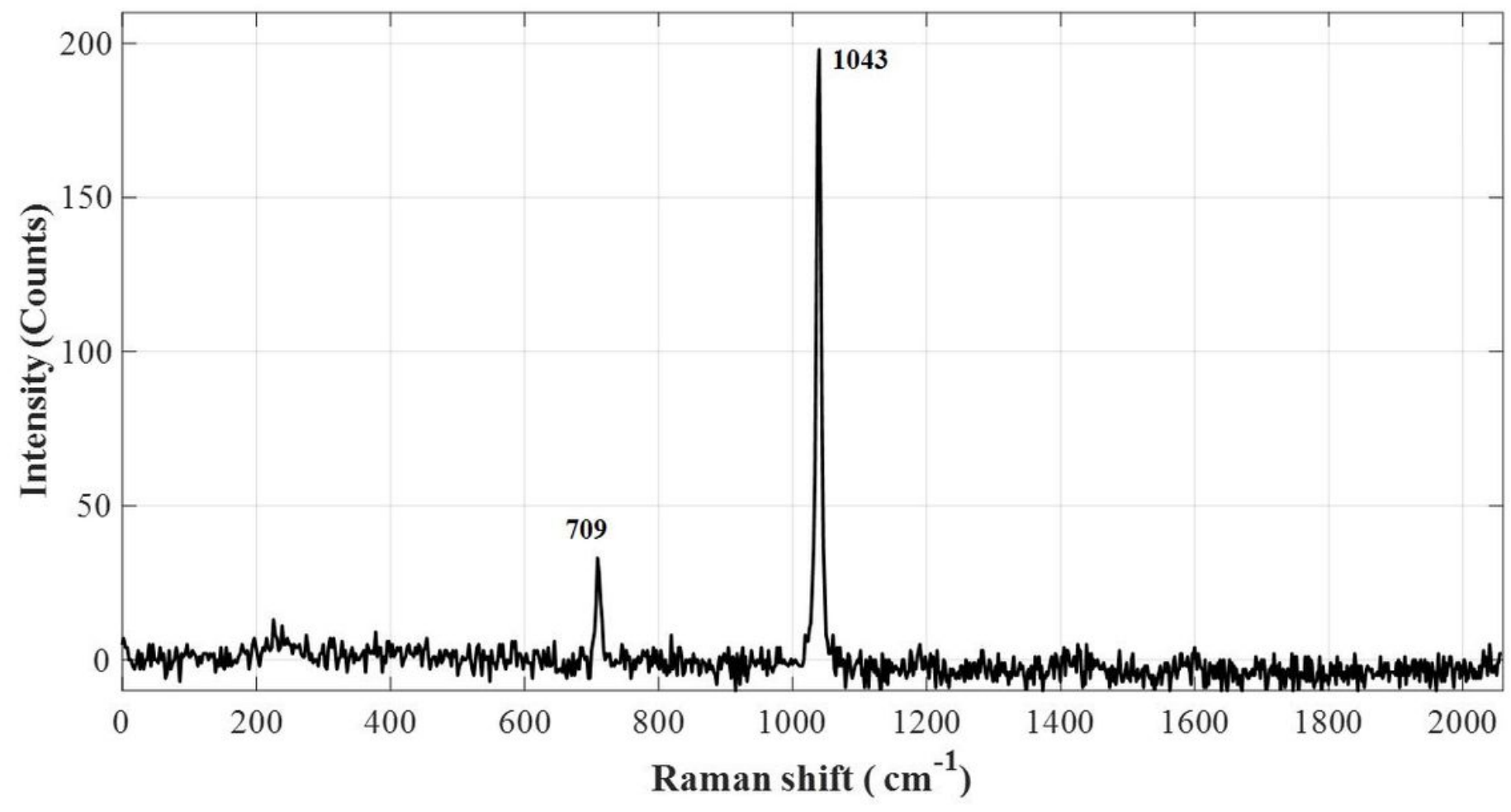

Figure 13

The measured Raman spectrum of the of Ammonium Nitrate sample. 


\section{Set Matching Sensitivity}
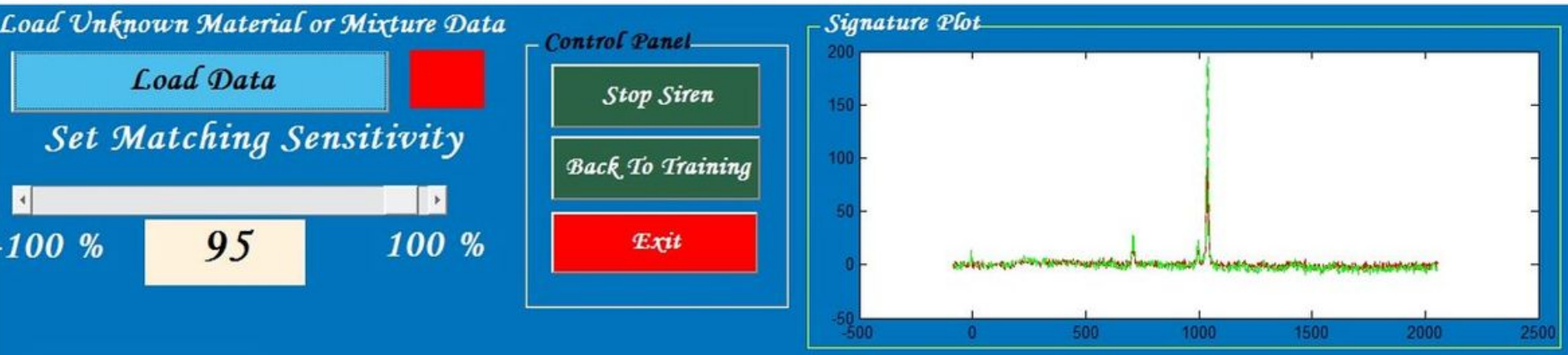

Identification Results

Material Expected Category

First Probability

Second Probability

Third Probability

\section{Explosive Material}

Material Expected Type

A $m m \mathfrak{N i t t}$
Matching Level

99.93\%

\section{Figure 14}

The recognition interface form shows that the material type is Ammonium Nitrate with matching level of $99.93 \%$.

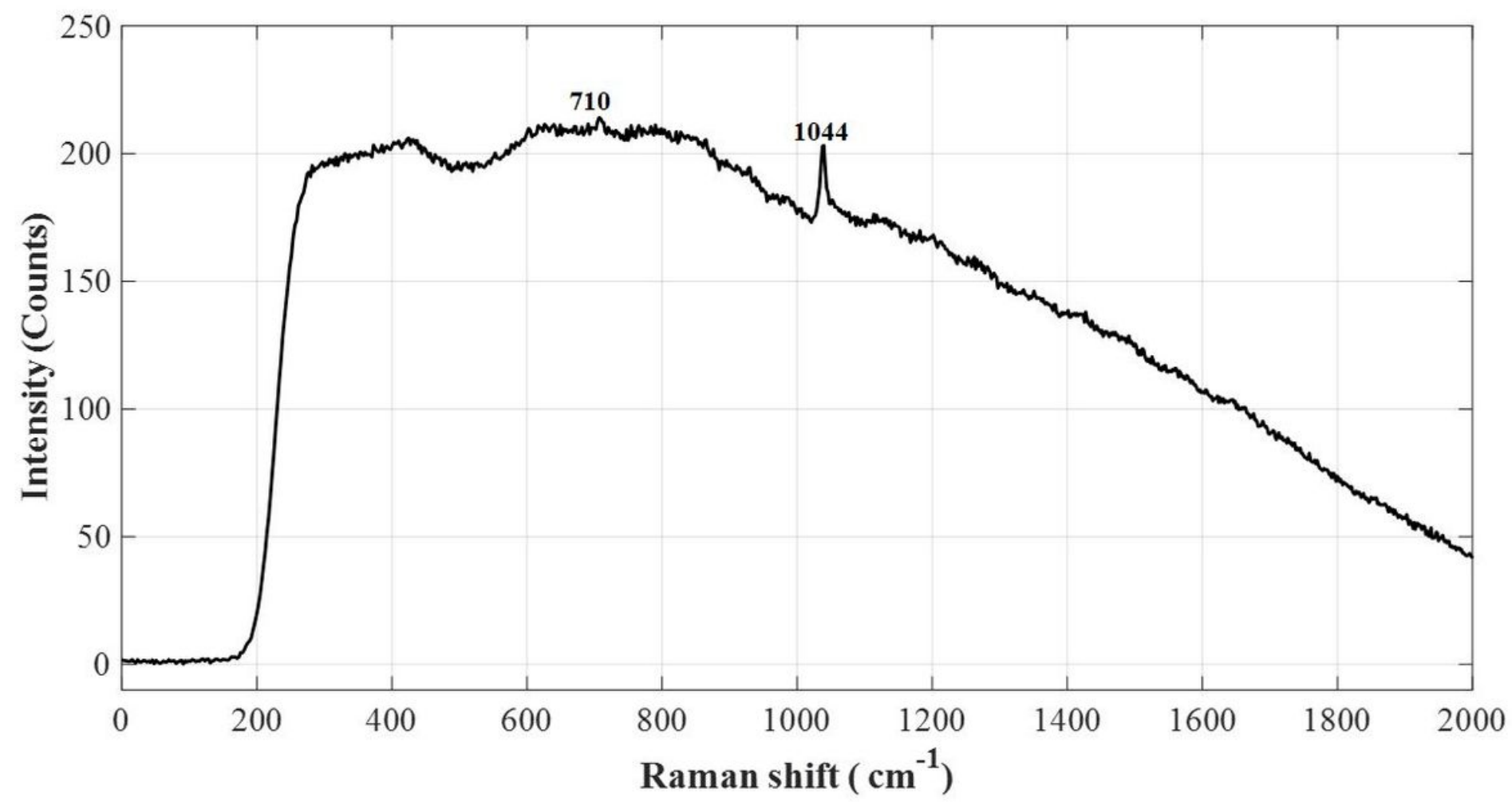

\section{Figure 15}

The measured Raman spectrum of the of Anfo sample. 


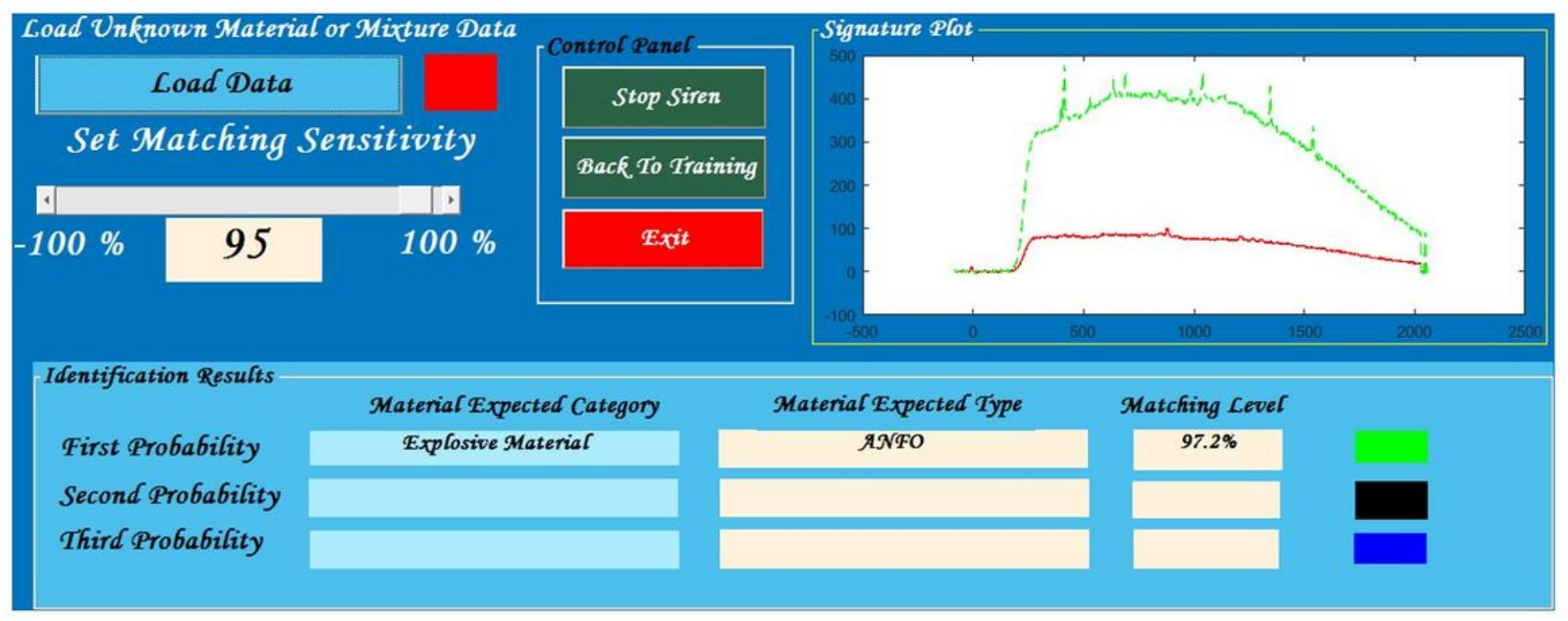

\section{Figure 16}

The recognition interface form shows that the material type is ANFO with matching level of $97.2 \%$.

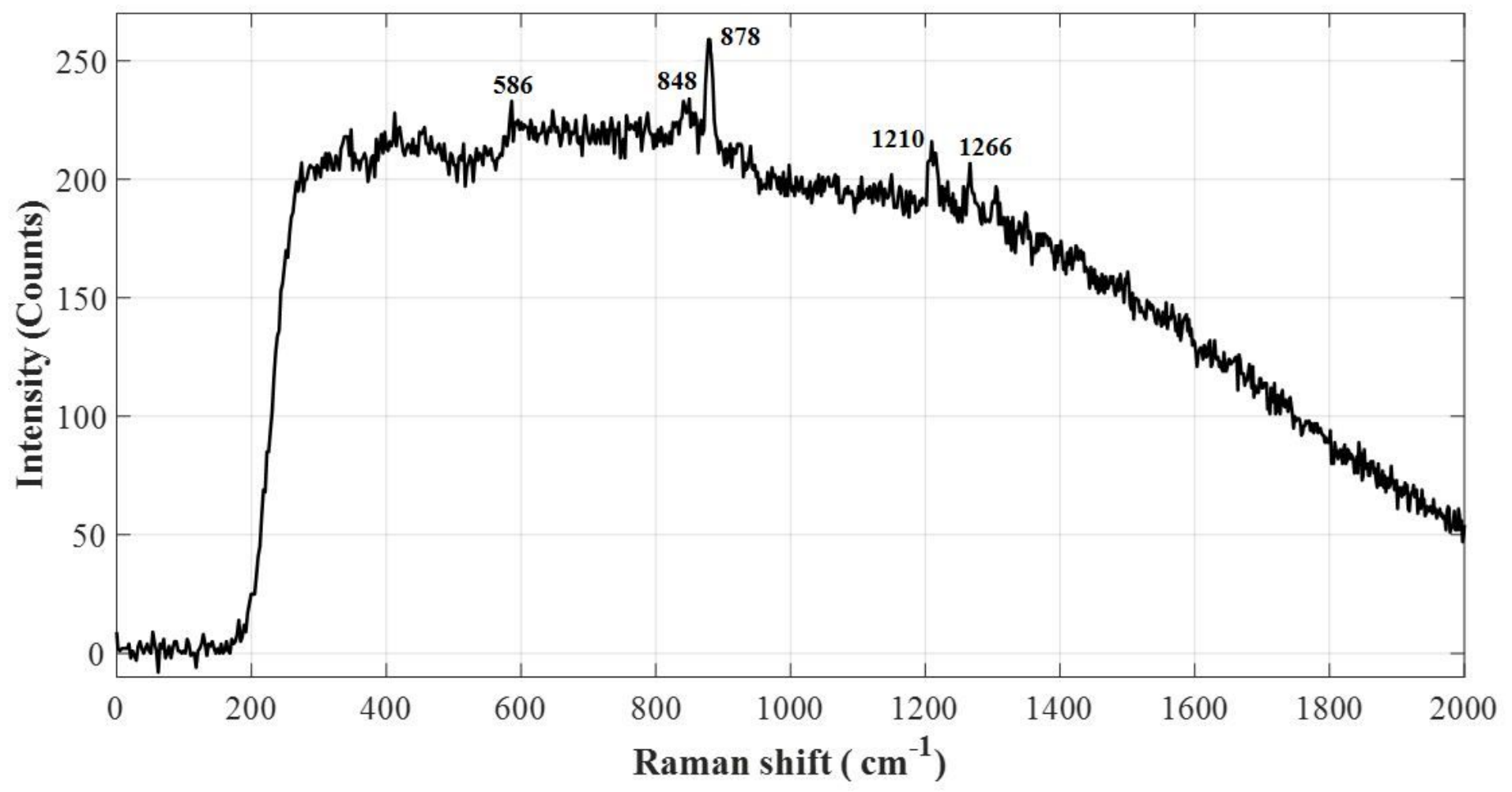

Figure 17

The measured Raman spectrum of the of RDX sample. 


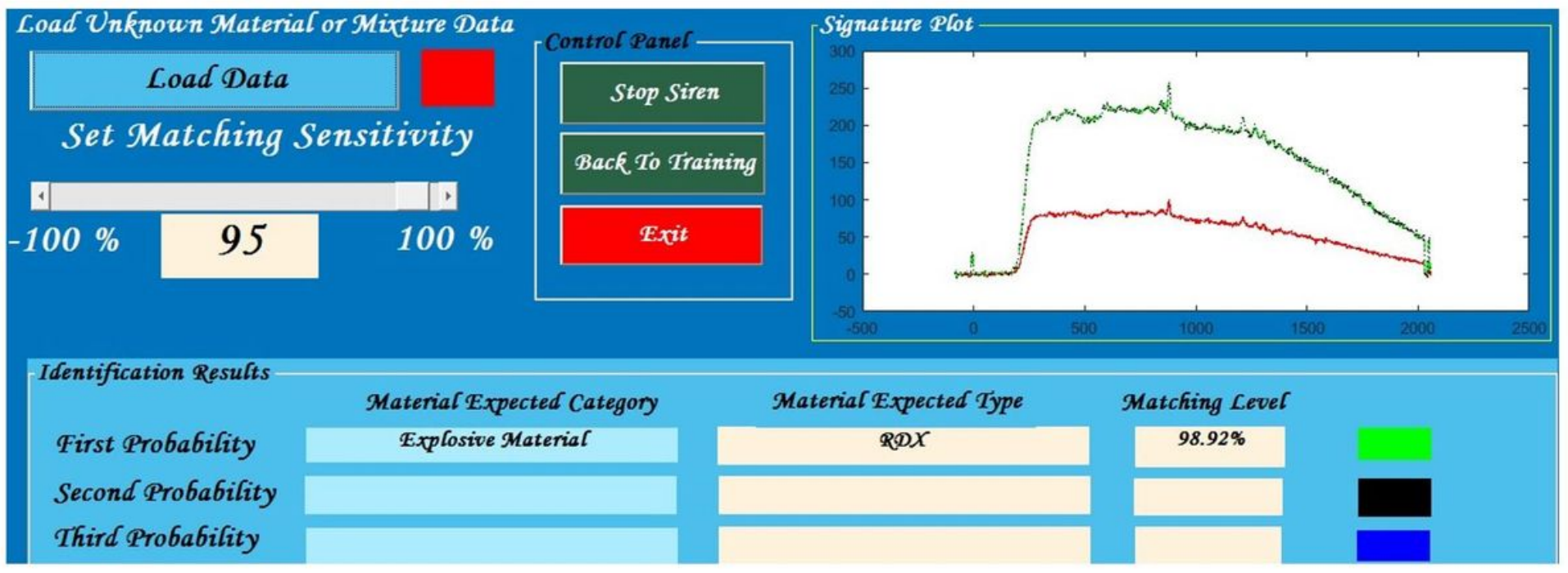

\section{Figure 18}

The recognition interface form shows that the material type is RDX with matching level of $98.92 \%$.

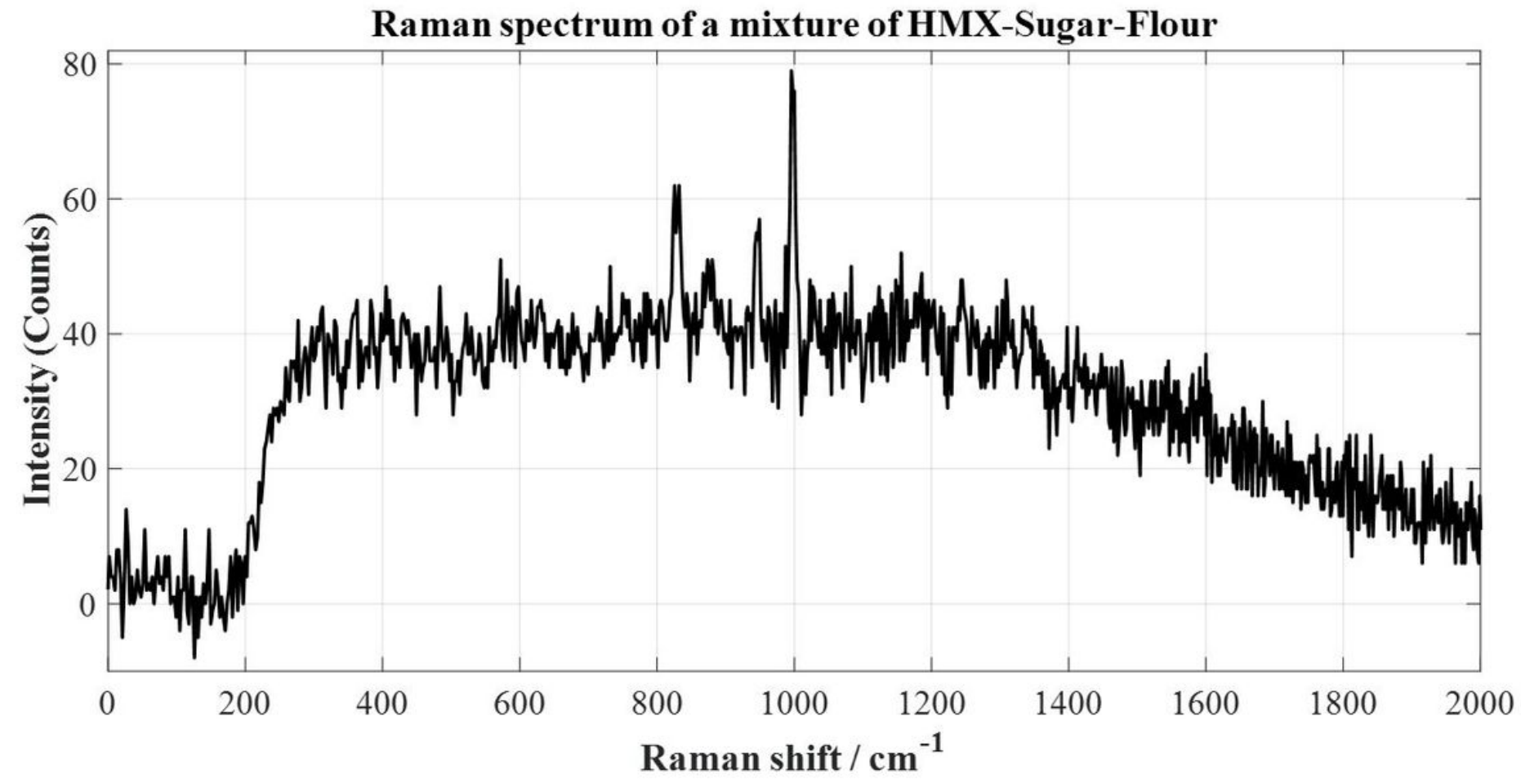

Figure 19

The measured Raman spectrum of the mixture $40 \% \mathrm{HMX}, 30 \%$ sugar and $30 \%$ flour. 


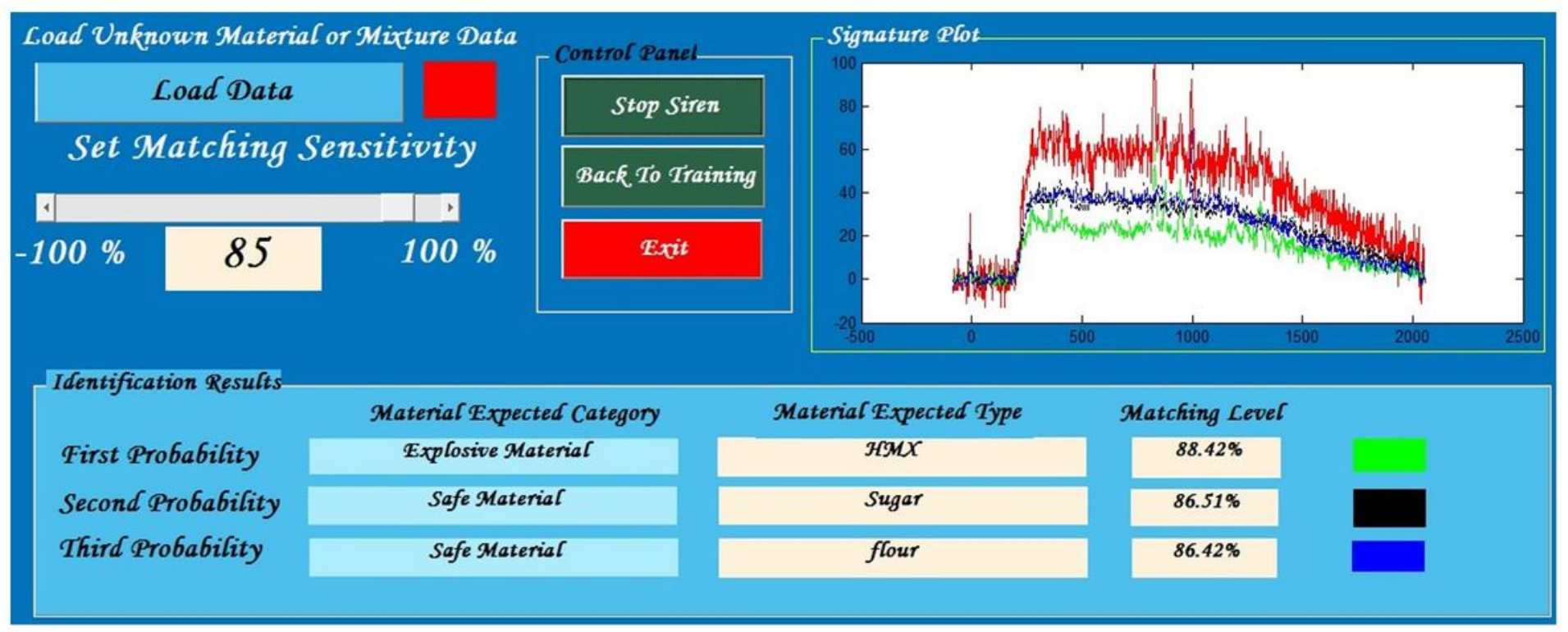

\section{Figure 20}

The recognition interface form shows the identified substances, the classification, the signature plot (on the top right part of the figure), and the matching levels of the substances form the mixture.

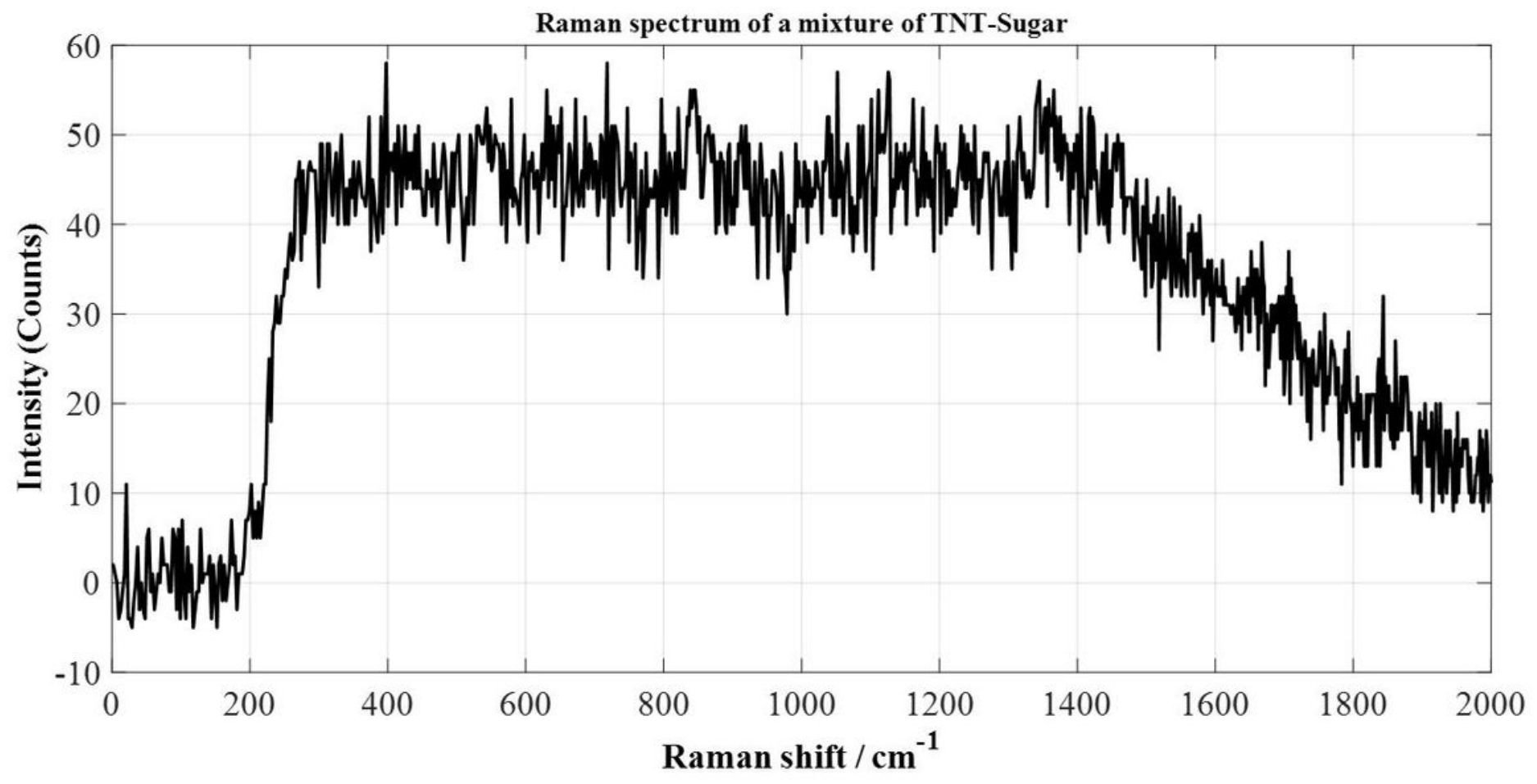

Figure 21

The measured Raman spectrum of the mixture of $50 \%$ of TNT, and $50 \%$ of sugar. 


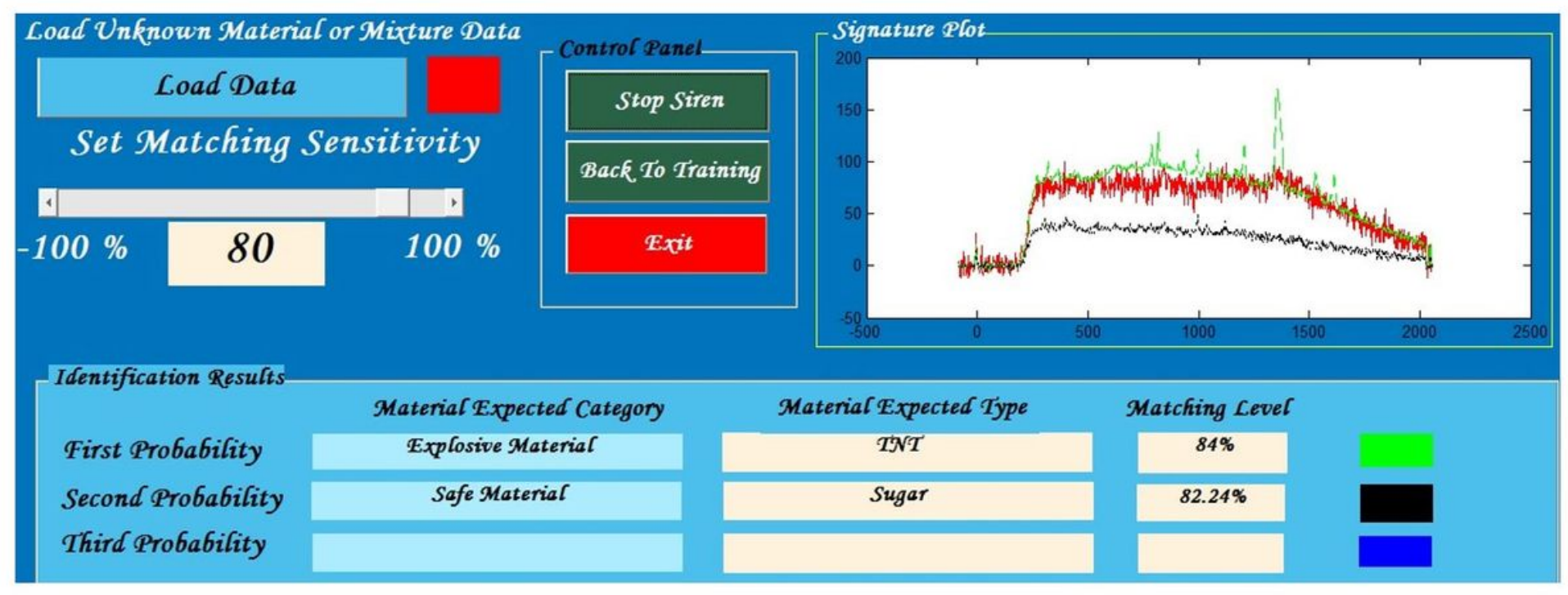

\section{Figure 22}

The recognition interface form shows that the system could identify, classify the mixture of TNT and the sugar with matching levels of $84 \%$, and $82.24 \%$, respectively. 